\section{PSICOLOGIA IBEROAMERICANA}

\section{Psicología lberoamericana}

ISSN: 1405-0943

psicología.iberoamericana@uia.mx

Universidad Iberoamericana, Ciudad de

México

México

García-Campos, Tonatiuh; García-y-Barragán, Luis Felipe Locus de Control y Tradicionalismo en el Estado de Guanajuato, México

Psicología Iberoamericana, vol. 19, núm. 1, enero-junio, 2011, pp. 72-79

Universidad Iberoamericana, Ciudad de México

Distrito Federal, México

Disponible en: http://www.redalyc.org/articulo.oa?id=133920896008

Cómo citar el artículo

Número completo

- Más información del artículo

- Página de la revista en redalyc.org

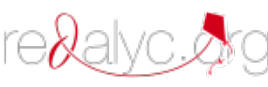

Sistema de Información Científica

Red de Revistas Científicas de América Latina, el Caribe, España y Portugal

Proyecto académico sin fines de lucro, desarrollado bajo la iniciativa de acceso abierto 


\title{
Locus de Control y Tradicionalismo en el Estado de Guanajuato, México
}

\author{
Locus of Control and Traditionalism in the State \\ of Guanajuato, México
}

Tonatiuh García-Campos

Luis Felipe García-y-Barragán*

Universidad de Guanajuato, Guanajuato, México

\section{RESUMEN}

Las atribuciones que podamos realizar sobre las causas de los eventos que nos suceden cotidianamente, nos llevan a definir si tenemos algún control sobre ellas o no. Considerando que las normas, creencias, valores y actitudes, reflejan la cultura subjetiva del grupo de pertenencia (Matsumoto, 1996), las atribuciones de control que puedan hacer las personas, estarán mediadas por el entorno sociocultural al que pertenecen, así como por la interiorización de las normas, valores y creencias del mismo (Díaz-Guerrero, 2003; García 2003). En esta investigación participaron 848 personas de cinco municipios del estado de Guanajuato, con una media de 28.58 años, siendo el $47.2 \%$ de ellos hombres. Los resultados muestran que la interiorización de la cultura tradicional afecta en el proceso de atribución del control; proceso que está asociado con la baja autoestima, motivación al logro, adherencias a tratamientos, entre otras.

Descriptores: Locus de control, Atribuciones, Tradicionalismo, Premisas Histórico-Socio-Culturales.

\section{ABSTRACT}

Attributions about the causes of the events in the everyday life give us the ability to define the extent of control about them. Considering that the norms, believes, values and attitudes are the reflex of the subjective culture from the group membership (Matsumoto, 1996), the control attributions that people can made are mediated by the interiorized norms, values and beliefs from the sociocultural context to which belongs (Díaz-Guerrero, 2003; García 2003). The sample was conformed by 848 participants from five municipalities of the State of Guanajuato in Mexico (Age mean = 25.58 years), 47.2\% of the sample were males. The result shows that the internalization of the traditional culture, affects the attribution process of control; process associated with the low self-esteem, achievement motivation, adhesions to treatments, among others.

Key Words: Locus of control, Attributions, Traditionalism, Historic-Socio-Cultural Premises

\footnotetext{
* Para correspondencia: Departamento de Psicología, Universidad de Guanajuato, Campus León Blvd. Puente Milenio \# 1001, Fracción del Predio San Carlos, Léon, Gto. CP 37670. Tonatiuh García Campos, tonatiuh@leon.ugto.mx. Tel: 014772674900 ext3644. Luis Felipe García y Barragán, psicosoc@hotmail.com. Tel: 01477 2674900, ext. 3611.
} 
Las atribuciones que se pueden realizar sobre las causas de lo que nos sucede cotidianamente, nos llevan a definir si tenemos algún control sobre ellas o no. Esto es a lo que Rotter (1966) ha denominado Locus de Control, el cual puede estructurarse en dos grandes factores: interno, cuando percibimos que tenemos la posibilidad de controlar lo que nos sucede (ej. habilidad de la persona) y; externo, cuando el control está depositado fuera de la persona, como podría ser la suerte o alguna deidad. Así, el Locus de Control (LC) se define como la creencia que uno tiene respecto a quién o qué factores controlan la vida (La Rosa, 1986; Lefcourt, 1991).

Al respecto, Lefcourt, Von Baeyer, Ware y Cox (1979, en Lefcourt, 1991) plantean un modelo de estudio del LC, multidimensional y multiatribucional de causalidad, en el que, basados en el control interno y externo, se desprenden dos áreas de aplicabilidad: el logro y la afiliación. También mencionan la importancia que puede tener si dentro de estas dos áreas se tiene éxito o fracaso en lo que se va a atribuir.

De esta forma, la creencia en la influencia de las capacidades y del esfuerzo de la persona en el mantenimiento de sus relaciones interpersonales, se considera como de control interno; por otro lado, quien considera el contexto y la suerte como responsable en la formación y mantenimiento de este tipo de relaciones, se le cataloga externo, en el ámbito de la afiliación.

La importancia de esta característica de personalidad la podemos encontrar en las publicaciones que se han hecho respecto al constructo en diferentes áreas de la salud y sociales. Por ejemplo, Alvarado (2003) reporta una relación del Locus de Control interno con una vida más satisfactoria, reducción de la mortalidad y una vejez satisfactoria. De igual forma, se ha relacionado el Locus de Control con la autoeficacia política y con las creencias sobre el control (Darmofal, 2010), con la satisfacción profesional; con efectos directos y como mediador parcial en madres de familia que trabajan (Noor, 2002). Laborin y Vega (2000) encontraron que las personas que se perciben como más satisfechas con su vida, poseen un Locus de Control interno; mientras que Zdanowicz, Janne y Reynaert (2004) encontraron al comparar adolescentes sanos con enfermos que los primeros presentan mayor puntaje de locus de control interno de la salud.
Asimismo, las mujeres presentan una tendencia hacia la desesperanza aprendida (Manger \& Eikeland, 2000), y a un Locus de Control externo (Cairns, McWhirter, Duffy \& Barry, 1990). Por otra parte, hay estudios (Adame, Jonson \& Cole, 1989; Chubb, Fertman \& Ross, 1997; Sorlie \& Sexton, 2003) que sostienen que no hay diferencias significativas entre los sexos.

En esta búsqueda del efecto de diferentes variables en países, se ha encontrado (Berry, Poortinga, Segal \& Dasen, 1995; Matsumoto, 1996) que los estadounidenses y otros europeos tienden a tener un mayor Locus de Control interno, y los asiáticos (principalmente los japoneses) son más propensos a uno externo. Así, las personas que viven en países industrializados aparecen con más Locus de Control interno, que quienes viven en países en vías de desarrollo. Lester (1992) plantea que las diferencias socioeconómicas afectan al tipo de LC predominantemente en los individuos, donde un nivel más bajo tiende a estar más relacionado con un LC externo.

En estudios realizados en México, Díaz-Guerrero (1997) reportó que tanto el paso del tiempo dentro de las etapas del desarrollo, como el nivel educativo, afectan el LC de las personas, mostrándose más internas. Aunque García (2003) plantea que la internalidad del Locus de Control aumenta con la edad, estabilizándose en la adolescencia.

García y Reyes (2000), partiendo en la propuesta de Lefcourt (1991) reportan que la estructura del LC para los mexicanos se compone de cinco factores; el primero denominado externo, se refiere a aquellas situaciones tanto de logro como de afiliación, cuyas atribuciones se hacen a factores externos. El segundo factor, logro interno, redacta situaciones de logro cuyas causas provienen de acciones directas o atribuciones propias de la persona. El tercero, el cual fue llamado social afectivo, se refiere a la obtención de metas, gracias a las relaciones afectivas de la persona. El cuarto factor, llamado afiliación con locus interno, menciona situaciones en las que el individuo se atribuye a sí mismo las razones por las cuales tiene buenas relaciones interpersonales, y por último, el factor statu quo familiar, que se refiere principalmente a situaciones de integración familiar causadas por la persona.

Con esta idea, diferentes investigaciones (García \& Corral-Verdugo, 2010; García \& Reyes, 2000) han 
reflejado el cambio de la tendencia a atribuir el control de manera interna. Esta característica no se presenta ba de dicha manera, en estudios anteriores, como el de La Rosa (1986), quien refleja a un mexicano que atribuye principalmente, de manera externa.

De acuerdo con Heine (2010), la forma en que los individuos manejan sus necesidades y deseos se encuentra moldeada por las teorías y concepciones que tienen del funcionamiento del mundo, y en específico de sus ideas, sobre el control que puedan tener sobre el ambiente que les rodea. Es así, que al considerar que las normas, creencias, valores y actitudes que se tienen, reflejan la cultura subjetiva del grupo de pertenencia (Matsumoto, 1996), las atribuciones de control que puedan hacer las personas, estarán mediadas por el entorno sociocultural al que pertenecen (Díaz-Guerrero, 2003; García 2003), así como a la interiorización de las normas, valores y creencias del mismo. Por ejemplo, García-Campos (2008) reporta el efecto del tradicionalismo en la personalidad, en específico en la instrumentalidad-expresividad.

Ahora, se considera que una sociocultura es el conjunto de afirmaciones utilizadas y aprobadas consistentemente por la mayoría de sus integrantes; en ese sentido, las Premisas Histórico-Socio-Culturales (PHSC's) de Díaz Guerrero (1997) se presentan como una buena estrategia para evaluar la internalización de la cultura y su efecto en el LC.

Considerando los constructos asociados con el LC y sus implicaciones, surgen diferentes incógnitas al respecto. De inicio, es conocer si esta tendencia interna que se reporta en los mexicanos, continúa y se presenta en muestras que no han sido consideradas en estudios previos. Adicionalmente, si la sociocultura de las personas afecta en los comportamientos de las mismas, incluso en la personalidad de los que han sido socializados dentro de ella, es de esperarse que la interiorización de las normas culturales o el alejamiento de ellas tenga un efecto en la percepción del control de lo que nos sucede.

\section{MÉTODO}

\section{Participantes}

Se eligieron 848 personas de cinco municipios del estado de Guanajuato, cuya edad fluctuó entre 15 y 58 años, con una media de 28.58 años y una desviación estándar de 11.22. La moda de escolaridad refleja bachillerato incompleto. El 47.2\% de la muestra fueron hombres.

\section{Instrumento}

Se utilizó la versión corta de la "escala multifactorial de Locus de Control" propuesta por García y Reyes (García 2003), que consta de 40 reactivos tipo Likert pictórica de siete opciones; se estructura de cinco factores (consistencia interna de $\alpha=.88$ ).

A continuación se presentan los factores en los que consiste el instrumento con el número de reactivos que se miden y su consistencia interna:

Externo. Factor que refiere atribuciones a elementos externos como causantes de nuestros resultados (Dios, la suerte, el destino, etcétera). Se mide con 16 reactivos y su consistencia interna es de alfa $=.85$.

Logro interno. Habla de los logros principalmente laborales y escolares; las atribuciones se deben a elementos internos de la persona (capacidades, inteligencia, esfuerzo, etc.). Consta de ocho reactivos, la consistencia es $\alpha=.91$.

Afiliación con locus interno. Referente a elementos internos como el esfuerzo que el individuo atribuye para lograr tener buenas relaciones interpersonales. Tres reactivos con alfa $=.65$.

Adicionalmente se contemplan dos indicadores, debido a su importancia teórica:

Social afectivo. Se refiere a atribuciones de logro, gracias a simpatía o lo agradable que sea la persona. Le corresponde dos reactivos $\alpha=.79$.

Statu Quo familiar. Se relaciona con que la familia de la persona se mantiene unida, gracias a deseos y esfuerzos que el individuo realiza. Dos reactivos con una consistencia interna de .73.

También se aplicó la versión corta de la escala de Premisas Histórico Socioculturales (PHSC's) de García y Reyes (2003), la cual es autoaplicable, consta de 42 reactivos tipo Likert con seis opciones de respuesta que van de totalmente de acuerdo a totalmente en desacuerdo. Los siete factores de la escala para población guanajuatense explican el 50.59\% de la varianza y tiene un índice de confiabilidad de .86. Los factores son los siguientes: 
Machismo. Se refiere a la superioridad del hombre (nueve reactivos con .77 de consistencia interna).

Respeto sobre amor. Se refiere a la mayor importancia que se le da al respeto a los padres, sobre el amor a los mismos (cuatro reactivos con .87 de consistencia interna).

Virginidad. Habla sobre la importancia de la virginidad prematrimonial de las mujeres (cinco reactivos con .71 de consistencia interna).

Obediencia afiliativa. La obediencia que debe de haber hacia los padres por ser padres (cuatro reactivos con .71 de consistencia interna).

Temor a la autoridad. Temor que los hijos sienten hacia sus padres (tres reactivos con .62 de consistencia interna).

Adicionalmente, se consideraron dos indicadores, debido a su relevancia teórica:

Dinámica cultural. Se refiere al cambio en donde los hijos no siempre deben obedecer a sus padres (dos reactivos con .68 de consistencia interna).

Reproducción social. Los hijos quieren ser como sus padres (dos reactivos con .64 de consistencia interna).

\section{Procedimiento}

Se aplicaron los instrumentos en áreas de trabajo, escuelas y espacios públicos, bajo el consentimiento informado de las autoridades (en su caso) y de los participantes. Las aplicaciones se hicieron de manera grupal e individual.

Para tener el índice de tradicionalismo de las personas, se hizo una sumatoria de los factores de las PHSC's que reflejan este factor (todos menos dinámica cultural); de esa sumatoria, se dividió en tres la muestra, arrojando tres grupos de tradicionalismo (bajo, medio y alto).

\section{RESULTADOS}

Para poder identificar si en el estado de Guanajuato, la tendencia hacia el control interno también se presenta, nos dimos a la tarea de identificar su perfil. Como se puede observar en la Figura 1, todos los factores (excepto Externo) tienen valores superiores a la media teórica (4). Lo anterior indica que efectivamente la tendencia interna en las atribuciones, se presenta en esta muestra y en este periodo histórico. Sin embargo, no hay que dejar de mencionar que el factor Social afectivo tiene una naturaleza externa y también obtiene valores relativamente elevados.

Figura 1. Perfil del locus de control de la muestra total

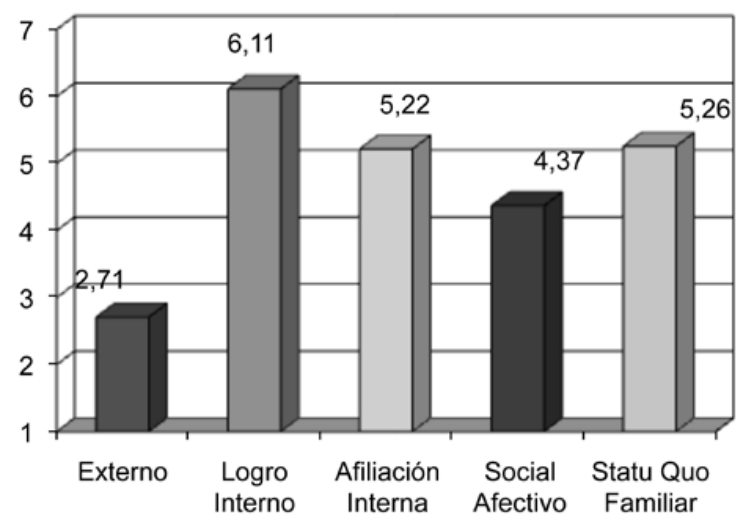

Como se ha mencionado en la literatura, los años de estudio afectan el tipo de atribución que puedan hacer las personas. Con base a estos hallazgos, nos dimos a la tarea de ver si este factor afecta el locus de control. Para ello, se dividió la muestra en cuatro grupos, aquellos que no habían cursado ningún año de estudios, hasta los que concluyeron ocho años; el siguiente grupo abarcó entre nueve y 11 años de estudio; el tercero, de 12 a 14 años y la última agrupación quedó conformada por las personas que tenían al menos 15 años de estudio. Lo anterior responde a niveles mínimos de primaria, secundaria, medio superior y superior, respectivamente.

Basados en estas agrupaciones, se puede observar (Tabla 1) que los puntajes responden de alguna manera al perfil antes presentado, sin embargo, podemos notar que a mayor número de años estudiados decrecen las atribuciones externas, internas en la afiliación y sociales afectivas. 
Tabla 1. Medias y diferencias en el Locus de Control por años cursados

\begin{tabular}{|c|c|c|c|c|c|c|}
\hline & $\begin{array}{l}\text { Escolaridad } \\
\text { en años }\end{array}$ & Media & DE & $F$ & gl & Prob. \\
\hline Externo & $\begin{array}{l}0 \\
9-11 \\
12-14 \\
15 \\
\text { Total }\end{array}$ & $\begin{array}{l}3.719 \\
2.699 \\
2.606 \\
2.181 \\
2.690\end{array}$ & $\begin{array}{l}1.448 \\
1.226 \\
1.279 \\
1.045 \\
1.331\end{array}$ & 34.135 & 3,706 & .000 \\
\hline Logro Interno & $\begin{array}{l}0 \\
9-11 \\
12-14 \\
15 \\
\text { Total }\end{array}$ & $\begin{array}{l}6.148 \\
6.036 \\
6.087 \\
6.198 \\
6.112\end{array}$ & $\begin{array}{l}.916 \\
.908 \\
.897 \\
.723 \\
.867\end{array}$ & 1.107 & 3,778 & .345 \\
\hline $\begin{array}{l}\text { Afiliación } \\
\text { Interna }\end{array}$ & $\begin{array}{l}0 \\
9-11 \\
12-14 \\
15 \\
\text { Total }\end{array}$ & $\begin{array}{l}5.606 \\
5.368 \\
5.099 \\
5.084 \\
5.227\end{array}$ & $\begin{array}{l}1.336 \\
1.357 \\
1.362 \\
1.326 \\
1.361\end{array}$ & 5.522 & 3,782 & .001 \\
\hline Social & $\begin{array}{l}0 \\
9-11 \\
12-14 \\
15 \\
\text { Total }\end{array}$ & $\begin{array}{l}5.198 \\
4.554 \\
4.209 \\
3.877 \\
4.365\end{array}$ & $\begin{array}{l}1.525 \\
1.581 \\
1.615 \\
1.634 \\
1.652\end{array}$ & 19.72 & 3,823 & .000 \\
\hline Statu Quo & $\begin{array}{l}0 \\
9-11 \\
12-14 \\
15 \\
\text { Total }\end{array}$ & $\begin{array}{l}5.996 \\
5.500 \\
4.814 \\
5.362 \\
5.250\end{array}$ & $\begin{array}{l}1.433 \\
1.604 \\
1.768 \\
1.401 \\
1.660\end{array}$ & 19.70 & 3,811 & .000 \\
\hline
\end{tabular}

Las diferencias que se presentan en los factores respecto a los años de estudio, están dadas en el factor Externo, por el primer grupo en comparación con el resto; de igual manera el grupo de mayor escolaridad con todos los demás. En la Afiliación interna es el grupo 1 (0-ocho años) el que presenta diferencias con los grupos 3 y 4 (mayores estudios).

En lo que respecta al factor Social afectivo, de nuevo el primer grupo se diferencia de los demás, así como el de mayores estudios con los dos con menor educación. Finalmente, en el factor Statu Quo familiar, el grupo equivalente al nivel medio superior (12-14 años de estudio), es diferente al resto de los grupos, así como el grupo 1 se distingue también de las personas con 12 o más años de estudio.
De acuerdo con las inquietudes que originaron la presente investigación, restaría conocer si el nivel de tradicionalismo sociocultural de las personas afecta su manera de atribuir el control de las cosas que les suceden. Para ello, como se mencionó anteriormente, se clasificó a la muestra de acuerdo a su nivel de tradicionalismo, generando tres grupos (Bajo, Medio y Alto). 
Tabla 2. Medias y diferencias en el Locus de Control tradicionalismo

\begin{tabular}{|c|c|c|c|c|c|c|}
\hline LC & / Tradicionalismo & Media & $\mathrm{DE}$ & $\mathbf{F}$ & gl & Prob. \\
\hline \multirow{3}{*}{ Externo } & Bajo & 2.15 & 1.06 & 66.62 & 2,646 & .000 \\
\hline & Medio & 2.44 & 1.14 & & & \\
\hline & Alto & 3.44 & 1.40 & & & \\
\hline \multirow{3}{*}{$\begin{array}{l}\text { Logro } \\
\text { Interno }\end{array}$} & Bajo & 6.14 & .776 & & & N.S. \\
\hline & Medio & 6.12 & .863 & & & \\
\hline & Alto & 6.05 & .937 & & & \\
\hline \multirow{3}{*}{$\begin{array}{l}\text { Afiliación } \\
\text { Interna }\end{array}$} & Bajo & 5.03 & 1.35 & 10.68 & 2,714 & .000 \\
\hline & Medio & 5.10 & 1.40 & & & \\
\hline & Alto & 5.55 & 1.21 & & & \\
\hline \multirow{3}{*}{$\begin{array}{c}\text { Social } \\
\text { Afectivo }\end{array}$} & Bajo & 3.99 & 1.61 & 18.45 & 2,743 & .000 \\
\hline & Medio & 4.19 & 1.61 & & & \\
\hline & Alto & 4.82 & 1.56 & & & \\
\hline \multirow{3}{*}{$\begin{array}{c}\text { Statu Quo } \\
\text { Familiar }\end{array}$} & Bajo & 4.89 & 1.59 & 14.41 & 2,729 & .000 \\
\hline & Medio & 5.16 & 1.66 & & & \\
\hline & Alto & 5.67 & 1.62 & & & \\
\hline
\end{tabular}

Los resultados nos muestran que la interiorización de la cultura tradicional afecta en el proceso de atribución del control. Como se muestra en la Tabla 2, quitando el caso del Logro interno, a mayor tradicionalismo se hacen atribuciones más externas (Externo y Social afectivo), aunque también se observa que las personas se atribuyen cierto control para mantener sus relaciones afiliativas y familiares. En el caso de las atribuciones externas (Externo y Social afectivo), las diferencias se presentan entre todos los grupos; caso contrario, en los factores Afiliación interna y Statu Quo familiar, éstas las produce el grupo más tradicional, en contraste con los dos restantes.

\section{DISCUSIÓN}

Como se pudo observar en los datos antes presentados, la tendencia del LC de esta muestra se mantiene en la misma dirección que los datos reportados por García y Reyes (2000) y por Góngora y Reyes (2000), en contraposición a lo reportado por La Rosa (1986) hace más de dos décadas. Lo anterior refleja que al pasar del tiempo y sin importar las latitudes, el Locus de Control interno es lo que caracteriza a los mexicanos, incluyendo a los guanajuatenses.

Sin embargo, hay que reconocer que el factor Social afectivo tiene un carácter externo y obtiene valores superiores a la media teórica, lo que refleja un aspecto cultural muy importante, como lo es la simpatía y la cohesión grupal que el colectivismo denota. Así, si trato de ser agradable (exigencia cultural), podré lograr las cosas que me propongo.

Como lo mencionó Díaz-Guerrero (1997), la educación es un agente de cambio, lo que implica que al tener mayores años de estudio ha demostrado que afecta en el tipo de atribuciones que se realizan. De igual manera, nuestros datos reflejan el impacto que tiene la educación formal en el LC. Apoyando la tesis de que a mayor número de años de estudio, el control interno se incrementa y, el externo presenta un decremento. Lo anterior refleja que la educación formal ayuda en responsabilizar, y por ende, a que el individuo se atribuya la responsabilidad de sus actos. 
Partiendo de que la cultura tradicional mexicana, ésta socializa a sus integrantes de tal manera que sean obedientes (para que el control esté fuera de ellos), que le den un peso considerable a las opiniones externas, que quieran ser como sus padres han sido, entre otras; es que se consideró que tales características podrían afectar el LC. Como lo muestran los datos, las personas más tradicionales atribuyen externamente, con mayor frecuencia, que aquellas personas alejadas

\section{REFERENCIAS}

Adame, D. A., Johnston, T. C. \& Cole, S.P. (1989). Physical fitness, body image, and locus of control in college freshman men and women. Perceptual and Motor Skills, No. 68, 400-402.

Alvarado, R. (2003). El Rol del Locus de Control como Factor Moderador en Trastornos Ansiosos y Depresivos en Adultos Mayores. PSYKHE, Vol. 12(1) pág. 57-64.

Berry, Poortinga, Segal, \& Dasen. (1995). Cross-cultural Psychology. Research and Applications. Cambridge University Press.

Cairns, E; McWhirter, L; Duffy, U \& Barry, R. (1990). Stability of Self-Concept in Late Adolescence: Gender and situational effects. Personality and individual differences, No. 11, 937-944.

Chubb, N. H; Fertman, C .I. \& Ross, J. L. (1997). Adolescent Self-Esteem and Locus of Control: A Longitudinal Study of Gender and Age Differences. Adolescence, No. 32 (125), 113-129.

Darmofal, D. (2010). Reexamining the Calculus of Voting. Political Psychology, 31(2), 149-174. doi: 10.1111/j.1467-9221.2009.00754.x.

Díaz-Guerrero, R. (1997). Psicología del mexicano. México: Editorial Trillas.

Díaz-Guerrero, R. (2003). Bajo las garras de la cultura. México. Editorial Trillas.

Flores G., M., Cortés A., M., Góngora C., E., \& Reyes L., I. (2002). Premisas socioculturales: Entre la transición y la Permanencia. En Asociación Mexicana de Psicología Social (eds.). La Psicología Social en México, (Vol 9, pp. 560-573), México: AMEPSO.

García, C. H. \& Corral-Verdugo, V. (2010). La identidad social y el locus de control interno en habitantes de la tradición cultural. Estos datos coinciden con los presentados por García Campos (García, 2003; GarcíaCampos, 2008). Sin embargo, podemos notar que las atribuciones internas asociadas a las relaciones afiliativas son mayores mientras el tradicionalismo se incrementa. Lo anterior concuerda con estudios (DíazGuerrero, 2003; Flores, Cortés, Góngora y Reyes, 2002; Triandis, 2001), donde queda claro que este tipo de relaciones son muy importantes para la cultura.

pobres del sur del Nuevo León, México. Revista de Psicología Social, 25 (2), 231-239.

García, T. (2003). Cultura y subculturas: el mexicano y su diversidad. Tesis para obtener el grado de Doctor en Psicología. Universidad Nacional Autónoma de México.

García-Campos, T. (2008). Cultura tradicional y masculinidad feminidad. Revista Interamericana de Psicología, 42, 1, 59-68.

García, T. \& Reyes, I. (2000). Estructura del Locus de Control en México. La Psicología Social en México, Vol. VIII, 158-164.

Góngora, E. \& Reyes I. (2000). El enfrentamiento a los problemas y el Locus de Control. La Psicología Social en México, Vol. VIII, 165-172.

Heine, S. J. (2010). Cultural Psychology, en Fiske, S. T., Gilbert. D. T. y Lindzey, G. Handbook of Social Psychology. New Jersey: John Wiley \& Sons.

Laborin, J. F., \& Vega, JA. (2000). Bienestar subjetivo y su relación con locus de control y el enfrentamiento. $\mathrm{La}$ Psicología Social en México, Vol. VIII, 192-199.

La Rosa, J. (1986). Escalas de Locus de Control y Autoconcepto: Construcción y validación. Tesis inédita Doctorado en Psicología Social. Facultad de Psicología, Universidad Nacional Autónoma de México.

Lefcourt, H. M. (1991). Locus of control. Measures of Personality and Social Psychological Attitudes. San Diego California: Academic Press.

Lester, M. (1992). Cooperative/competitive strategies and locus of control. Psychological Reports, 71, 594-610.

Manger, T. \& Eikeland, O. (2000). On the relationship between locus of control, level of ability and gender. 
Scandinavian Journal of Psychology, Vol. 41, pág. 225229.

Matsumoto, D. (1996). Culture and Psychology. Brooks/ Cole Publishing Company.

Noor, N. (2002). Work-Family Conflict, Locus of Control and Women's Well Being: Tests of Alternative Pathways. The Journal of Social Psychology, 142(5), 645-662.

Rotter, J. B. (1966). Generalized expectancies for internal versus external control of reinforcement. Psychological Monographs, 80, No. 609.
Sorlie, T. \& Sexton, H. (2004). Predictors of change in health locus of control following surgical treatment. Personality and Individual Differences, 36(5), 9911004. doi: 10.1016/S0191-8869(03)00167-3.

Triandis, H. C. (2001). Individualism-collectivism and personality. Journal of Personality, 69, 907-924.

Zdanowicz, N.; Janne, P. \& Reynaert, Ch. (2004). ¿Juega el Locus de Control de la salud un papel clave en la salud durante la adolescencia? European Journal of Psychiatric, Vol. 18(2), 117-124. 